\title{
Utilidad del Doppler pulsado en el diagnóstico ecográfico de neumotórax oculto: a propósito de un caso
}

\section{Usefulness of pulsed Doppler in the Ultrasound Diagnosis of Occult Pneumothorax: A Case Report}

Recibido: 24 Abril 2020 | Aceptado: 16 Junio 2020

\author{
DAVID EnRIQUe MontaÑa MANRIQUea \\ Médico especialista en Medicina de Urgencias, \\ Pontificia Universidad Javeriana, Bogotá, Colombia \\ Leidy Jholeth Montero Vargas \\ Médica residente de Medicina de Urgencias, Pontificia \\ Universidad Javeriana, Bogotá, Colombia \\ Carlos Valencia \\ Médico especialista en Medicina de Urgencias, \\ Pontificia Universidad Javeriana, Bogotá, Colombia
}

a Autor de correspondencia: davidmontana@javeriana.edu.co

Cómo citar: Montaña Manrique DE, Montero Vargas LJ, Valencia C. Utilidad del Doppler pulsado en el diagnóstico ecográfico de neumotórax oculto: a propósito de un caso. Univ. Med. 2020;61(4). https:// doi.org/10.11144/Javeriana.umed61-4.dopp

\section{RESUMEN}

El neumotórax se define como una acumulación de aire entre la pleura parietal y visceral, secundaria a varias condiciones médicas; de ahí que la radiografía de tórax sea el método diagnóstico más utilizado para su detección. Sin embargo, en el caso de neumotórax oculto es poco sensible; mientras que la tomografía de tórax representa el método más sensible y específico para su diagnóstico. Así mismo, se ha demostrado que el ultrasonido a la cabecera del paciente tiene rendimientos similares a la tomografía de tórax y una sensibilidad superior con relación a la radiografía de tórax. Recientemente, se describió una nueva técnica para evaluar el deslizamiento pulmonar utilizando el Doppler pulsado (PW), bajo el principio que el movimiento del parénquima pulmonar se pierde junto con la señal Doppler del área. El artículo presenta el caso de una paciente que consulta por dificultad respiratoria posterior a un accidente de tránsito, donde la radiografía de tórax y los modos habituales de ultrasonido no permitieron diagnosticarla; en tanto que la realización del PW sugirió la presencia de neumotórax, lo que posteriormente se confirmó con la tomografía de tórax. Por ello, el modo PW se podría incluirse como complemento al algoritmo de la ecografía pulmonar para descartar la presencia de neumotórax, a fin de mejorar su rendimiento, por ser un método fácil y rápido de realizar con el cual podemos efectuar un diagnóstico preciso.

Palabras clave

ultrasonografía Doppler; neumotórax; medicina de emergencia; evaluación enfocada con sonografía para el trauma.

\section{ABSTRACT}

Pneumothorax is defined as a collection of air between parietal and visceral pleural layers, which can be caused by different medical conditions, being the chest radiography the most used medical diagnostic tool for its detection, but with a low performance in case of 
hidden pneumothorax, where Chest Tomography has an important role representing the most sensitive and specific method of diagnosis. Likewise, it has been demonstrated that bedside Ultrasound has similar performances to chest tomography and a superior sensitivity compared to chest radiography. Recently, a new technique was described to evaluate lung sliding by using Pulsed Doppler Wave or Pulsed Wave (PW), since movement of lung parenchyma is lost along with Doppler signal from the area. Here we present the case of a patient who consults for respiratory distress after a traffic accident, where the chest radiography and the usual modes of ultrasound did not allow the diagnosis to be made, while the performance of PW suggested the presence of pneumothorax, which was subsequently confirmed by chest tomography, considering PW mode could be a complement to the lung ultrasound algorithm to rule out the presence of pneumothorax in order to improve its performance, being an easy and quick method to perform, allowing an accurate diagnosis.

Keywords

Doppler ultrasonography; pneumothorax; emergency medicine; focused assessment with sonography of trauma.

\section{Introducción}

El neumotórax es una condición patológica caracterizada por una acumulación de aire entre la pleura parietal y la visceral. Su sintomatología es diversa y con un amplio espectro de presentación, que varía desde síntomas leves o incluso asintomáticos hasta complicaciones potencialmente fatales. Puede tener diferentes etiologías como trauma, iatrogenia (tras la inserción de un catéter venoso central, ventilación mecánica, toracentesis, biopsias pulmonares, entre otras), e incluso ocurrir de manera espontánea (1). La radiografía de tórax es el método diagnóstico más usado, con una sensibilidad del $53 \%$ para esta entidad, y un menor rendimiento en algunos casos particulares como neumotórax oculto (2). Entre tanto, la tomografía de tórax representa el método más preciso para su diagnóstico, y es el patrón de referencia (gold standard); sin embargo, condiciona un gran número de desventajas, como lo son el traslado del paciente para el procedimiento, el costo, la demora para su toma y la irradiación (1).

Por otro lado, el ultrasonido ha demostrado una sensibilidad del $100 \%$ y una especificidad del $99 \%$, para el diagnóstico de neumotórax
(3, 4, 5). En los casos de neumotórax oculto, se ha documentado una sensibilidad y una especificidad del $92 \%$ y del $99,4 \%$, respectivamente, lo que ofrece una gran ventaja al ultrasonido, pues este muy superior a la radiografía de tórax $(2,6,7,8,9,10)$. Una de las ventajas del ultrasonido es su capacidad para evidenciar el movimiento pleural, que, en algunas ocasiones, es difícil de evaluar con los modos ecográficos tradicionales (modos B y M), que se dificulta más por características de la lesión o propias del paciente, como enfermedad pulmonar obstructiva crónica, abundante grasa, entre otras, por lo que se requiere la obtención de imágenes en otros modos $(11,12)$.

Hace poco se describió una nueva técnica de ultrasonido para evaluar el deslizamiento pleural por medio de la técnica de Doppler pulsado (PW). El PW identifica los cambios en la frecuencia de las ondas de ultrasonido para identificar el movimiento. Se diferencia de las demás técnicas, porque trabaja independientemente de la dirección del flujo y detecta movimiento con una mayor sensibilidad a expensas de la información de dirección y velocidad (incluso a baja velocidad). Por lo tanto, supone que en presencia de un neumotórax, el movimiento del parénquima pulmonar se pierde junto con la señal de ultrasonido del área; en este caso, el movimiento del tejido pulmonar lo representa una onda de flujo Doppler que puede ser positiva o negativa, y esta depende del ángulo del transductor, así como del ángulo de isoniación utilizado (12).

La ecografía se realiza con elevación de la cabecera de la cama a 45 grados, debido a la intolerancia al decúbito. Se coloca el transductor en el área más anterior del tórax (2-3 espacios intercostales) con línea media clavicular. De esta manera se obtienen imágenes de ambos hemitórax en modo B, M y PW. Para su uso, la ganancia del equipo Doppler debe ser ajustada en el lado no afectado. Se inicia con una ganancia baja y se incrementa gradualmente hasta que se detecte el movimiento pleural normal; luego, se evalúa el lado afectado. En presencia de neumotórax, no se evidenciará el movimiento de las láminas pleurales. Una limitación de esta 
técnica es que, por la alta ganancia, puede haber ruido ecográfico que se interprete como un falso movimiento de las pleuras $(11,12)$. A continuación, presentaremos un caso clínico que demuestra su utilidad e impacto en el proceso diagnóstico y terapéutico de los pacientes.

\section{Caso clínico}

El caso corresponde a una mujer de 60 años de edad con antecedente de dislipidemia, quien ingresó al departamento de urgencias 24 horas después de haber sido arrollada por una motocicleta mientras ella estaba en calidad de peatona. Acudió al servicio, donde se le identificó trauma craneoencefálico leve asociado a trauma cerrado de tórax y en sus extremidades. En principio, fue valorada extrainstitucionalmente y le suturaron una herida en el cuero cabelludo y le dieron egreso. Ante la persistencia de dolor en su tronco y dificultad respiratoria, decidió consultar nuestra institución.

En la revisión primaria no se le identificaron lesiones potencialmente fatales. Respecto a la revisión secundaria, se encontró a la mujer hipertensa (TA: 140/79 $\mathrm{mm} \mathrm{Hg}$ ), polipnéica (FR: 26 r. p. m.) e hipoxémica (saturación: 85 $\% ; \mathrm{FiO}_{2}: 21 \%$ ), y con frecuencia cardiaca normal (87 1.p.m.). En la auscultación, se documentaron crépitos en la base pulmonar derecha, dolor a la palpación de los primeros arcos costales posteriores derechos, y normal en el resto del examen físico. Se solicitó tomarle creatinina, que reportó ser normal; hemograma, que mostró leve leucocitosis $(11.000 / \mathrm{dL})$; gases arteriales, que indicaron un trastorno moderado de la oxigenación ( $\mathrm{PaFio}_{2}: 210 \mathrm{~mm} \mathrm{Hg} / \%$ ) sin trastorno de la ventilación, y radiografía de tórax posteroanterior, que fue normal (figura 1).

\section{Figura 1}

Radiografía de tórax en proyección posteroanterior normal

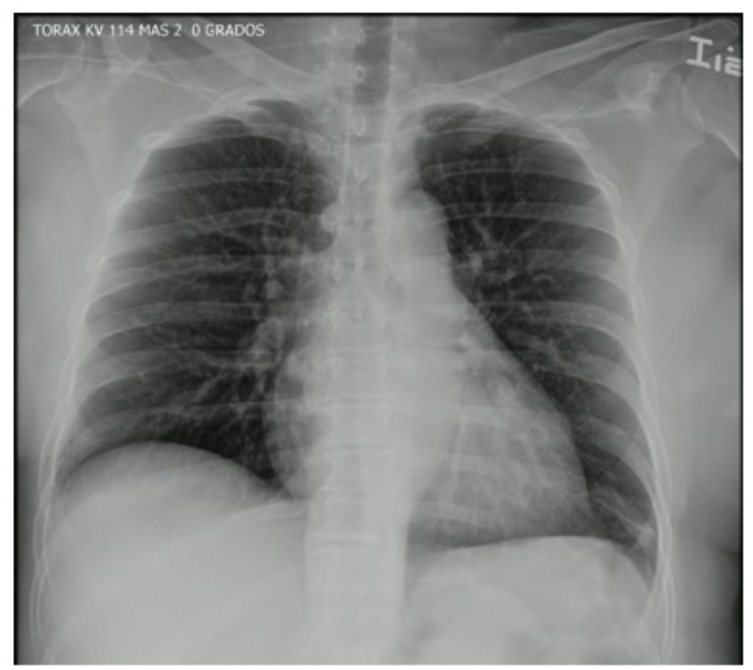

Ante la persistencia de la sintomatología, se consideró la toma de una ecografía pleural a la cabecera del paciente, con un transductor de alta frecuencia (10-15 MHz). En la ecografía, en los modos B (figura 2) y M (figura 3), se encontró deslizamiento pleural; al igual que el signo de playa y arena. Hasta ese momento se descartó la presencia de neumotórax. No obstante, al considerar los signos y los síntomas de la paciente, se decidió avanzar el estudio hacia la realización del modo PW, teniendo en cuenta su alto valor diagnóstico para el neumotórax oculto. En los hallazgos se observó la emisión de la señal asociada al movimiento del parénquima pulmonar en el hemitórax izquierdo, pero se perdía en el hemitórax derecho, con la subsecuente sospecha elevada de neumotórax (figura 4).

\section{Figura 2.}

Deslizamiento pulmonar en modo B: hallazgo normal en los dos campos pulmonares

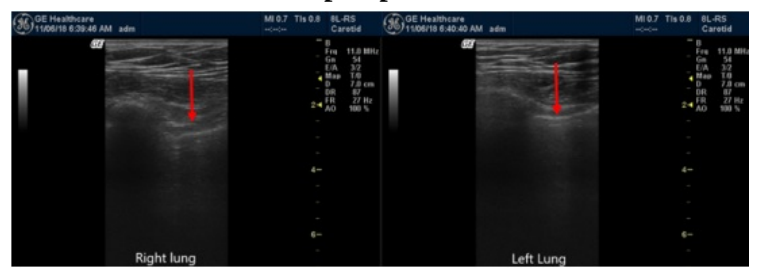


Figura 3.

Deslizamiento pulmonar en modo M y signo de playa y arena: hallazgo normal en los dos campos pulmonares

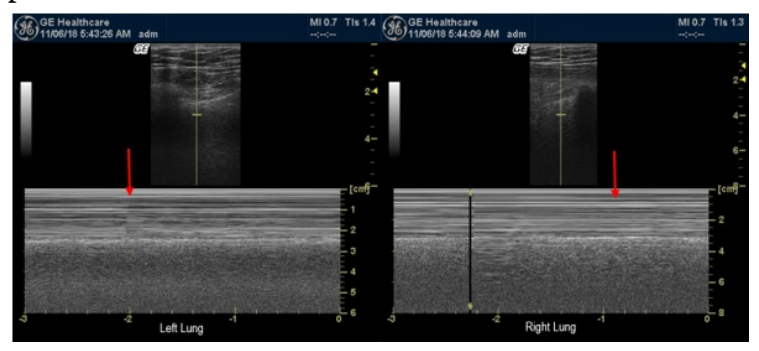

\section{Figura 4.}

Deslizamiento pulmonar en modo PW. El recuadro izquierdo corresponde al campo pulmonar izquierdo, en el cual está presente, y el recuadro derecho corresponde al campo pulmonar derecho, donde se encuentra ausente, debido al neumotórax

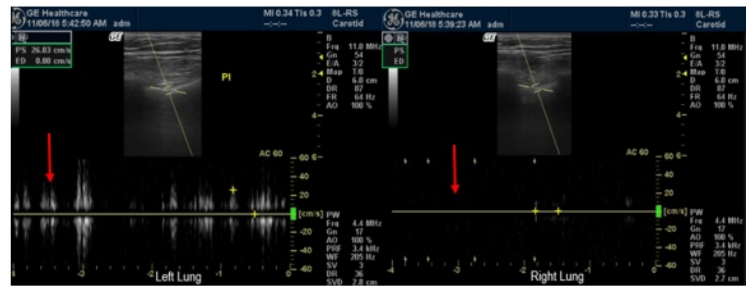

Teniendo en cuenta los hallazgos del ultrasonido, se decidió ampliar el estudio con una tomografía de tórax con contraste (figura 5). Con esta se confirmó la presencia de una pequeña cámara de neumotórax derecha con distancia interpleural en el ápice de $6 \mathrm{~mm}$, fractura impactada del aspecto dorsal del tercer arco costal derecho y fracturas laminares del aspecto posterolateral en el cuarto y quinto arcos costales derechos, asociados a escaso derrame pleural derecho y atelectasias subsegmentarias de ambos lóbulos inferiores.
Figura 5.

Tomografía de tórax y cámara de neumotórax derecho

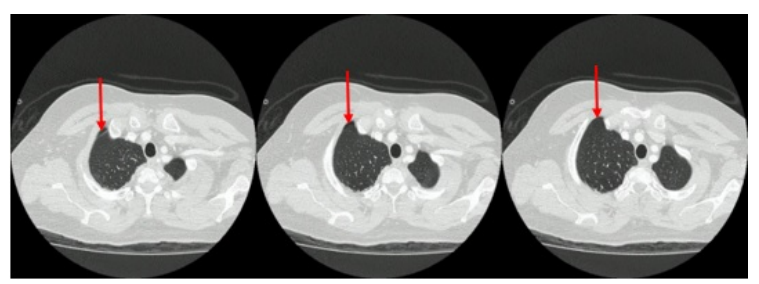

Con dichos hallazgos, la paciente fue llevada a toracostomía cerrada derecha. Permaneció en vigilancia clínica durante tres días con resolución satisfactoria del cuadro y se le retiró el tubo de toracostomía. Posteriormente, fue dada de alta sin complicaciones.

\section{Discusión}

La incidencia del neumotórax oculto es cercana al $5 \%$, según los registros de trauma (13). Aunque la ultrasonografía no suele utilizarse de modo rutinario, avances recientes en su técnica y tecnología han demostrado su capacidad para el diagnóstico en emergencias, siendo este un escenario donde el ultrasonido puede ser un método confiable y preciso para su detección $(11,14,15)$. Además, representa una ventaja en la práctica, pues se realiza a la cabecera del paciente, lo que disminuye la exposición a radiación ionizante generada por otros métodos diagnósticos para el paciente y especialmente para el operador $(16,17,18)$. También se ha señalado una mayor sensibilidad y especificidad que la radiografía de tórax $(6,7,8,9,10)$.

Incluso en la ecografía común, puede haber dificultades en la interpretación del deslizamiento pleural, sobre todo en los modos B o M. Como se evidencia en casos de atelectasias masivas, síndrome de dificultad respiratoria, adherencias pleurales, bulas o intubación selectiva, entre otras $(19,20)$. Es por esto por lo que la inclusión del modo PW para evaluar el movimiento del parénquima pulmonar, dentro del algoritmo diagnóstico del neumotórax, aportaría información útil para su detección. No obstante, se debe tener 
en cuenta que implica una mayor "curva de aprendizaje", pues es posible que haya errores en su interpretación, por una falla en la angulación del transductor (operador dependiente), si se dirige perpendicular al movimiento del pulmón, ya que no se detectaría el movimiento en este ángulo. Esto se corrige con un ligero "abaniqueo" del transductor.

Con la información revisada en la literatura y la experiencia en esta presentación de caso, se sugiere la técnica PW como una alternativa válida y precisa en la detección de neumotórax oculto, con un subsecuente impacto positivo en el pronóstico del paciente, facilidad de oportunidad y disminución en la radiación y de costos que se presentan, por ejemplo, en una tomografía computarizada. Sin embargo, se necesitan estudios adicionales que confirmen estos hallazgos con una mayor significancia estadística y que evalúen el rendimiento diagnóstico de los protocolos de ultrasonido en este contexto.

\section{Conclusiones}

El ultrasonido es una herramienta que ha logrado mayor acogida a medida que pasa el tiempo y se desarrollan nuevas técnicas para su uso. Ha demostrado ser muy eficaz en el servicio de urgencias para detectar múltiples patologías, dentro de las que se incluyen el neumotórax, ya que tiene muchas ventajas sobre otros métodos diagnósticos: es una herramienta de fácil uso, con una adecuada disponibilidad, portabilidad y repetibilidad; además de esto, nos aporta una apropiada sensibilidad y especificidad para su diagnóstico. Así, nos brinda un alto grado de confiabilidad para la detección temprana y oportuna del neumotórax y, con esto, lograr un tratamiento rápido y adecuado de esta patología. El modo PW es una herramienta que complementa los algoritmos ecográficos de detección de neumotórax; sin embargo, se deben ampliar los estudios para su uso.

\section{Agradecimientos}

Al Servicio de Urgencias del Hospital Universitario San Ignacio y al Grupo de Investigación en Ultrasonido de Urgencias de la Pontificia Universidad Javeriana.

\section{Referencias}

1. Kelly A-M, Weldon D, Tsang AYL, Graham CA. Comparison between two methods for estimating pneumothorax size from chest X-rays. Respir Med [internet]. 2006 [citado 2018 Dec 12];100:1356-9. Disponible en: https://www.resmedjournal.com/ar ticle/S0954-6111(05)00512-3/pdf

2. Rowan KR, Kirkpatrick AW, Liu D, Forkheim KE, Mayo JR, Nicolaou S. Traumatic pneumothorax detection with thoracic US: correlation with chest radiography and CTinitial experience. Radiology. 2002 Oct;225(1):210-4. https://doi.org/10.1 148/radiol.2251011102

3. Wilkerson GR, Stone MB. Sensitivity of bedside ultrasound and supine anteroposterior chest radiographs for the identification of pneumothorax after blunt trauma. Acad Emerg Med. 2010 Jan 1;17(1):11-7. https://doi.org/10.1111/j. 1553-2712.2009.00628.x

4. Moreno-Barrera RL, De la PortillaVillanueva MA. Determinación de la validez diagnóstica de la ecografía de tórax como prueba única para el diagnóstico de neumotórax postraumático comparada con la tomografía computada. An Radiol Méx. 2018;17(2):140-4. https://doi.org /10.24875/ARM.M18000022

5. Soldati G, Testa A, Sher S, Pignataro G, La Sala M, Silveri NG. Occult traumatic pneumothorax: diagnostic accuracy of lung ultrasonography in the 
emergency department. Chest. 2008 Jan 1;133(1):204-11. https://doi.org/10 $.1378 /$ chest.07-1595

6. Tataroglu O, Telli Erdogan S, Tayfur I, Ahmet Afacan M, Genc Yavuz B, Colak S, et al. Diagnostic accuracy of initiai chest $\mathrm{x}$-rays in thorax trauma. J Coll Physicians Surg Pakistan. 2018 Jul 1;28(7):546-8. https://doi.org/10.29 271/jcpsp.2018.07.546

7. Sartori S, Tombesi P, Trevisani L, Nielsen I, Tassinari D, Abbasciano V. Accuracy of transthoracic sonography in detection of pneumothorax after sonographically guided lung biopsy: Prospective comparison with chest radiography. Am J Roentgenol. 2007;188(1):37-41. https://doi.org/10. 2214/AJR.05.1716

8. Blaivas M, Lyon M, Duggal S. A prospective comparison of supine chest radiography and bedside ultrasound for the diagnosis of traumatic pneumothorax. Acad Emerg Med. 2005;12(9):844-9. https://doi.org /10.1197/j.aem.2005.05.005

9. Abdalla W, Elgendy M, Abdelaziz A, Ammar M. Lung ultrasound versus chest radiography for the diagnosis of pneumothorax in critically ill patients: a prospective, single-blind study. Saudi J Anaesth. 2016;10(3):265. https://doi. org/10.4103/1658-354X.174906

10. Alrajab S, Youssef AM, Akkus NI, Caldito G. Pleural ultrasonography versus chest radiography for the diagnosis of pneumothorax: review of the literature and meta-analysis [internet]. 2013 [citado 2018 Dec 16]. Disponible en: http://ccforum.com/con tent/17/5/R208

11. Cunningham J, Kirkpatrick AW, Nicolaou S, Liu D, Hamilton DR, Lawless B, et al. Enhanced recognition of "lung sliding" with power color Doppler imaging in the diagnosis of pneumothorax. J Trauma.
2002;52(4):769-71. https://doi.org/10. 1097/00005373-200204000-00029

12. Rose G, Siadecki S, Tansek R, Baranchuk N, Saul T. A novel method of assessing for lung sliding using Doppler imaging. Am J Emerg Med. 2017;35(11):1738-42. https://doi.org/1 0.1016/j.ajem.2017.09.006

13. Ball CG, Kirkpatrick AW, Feliciano DV. The occult pneumothorax: what have we learned? Can J Surg [internet]. 2009 [citado 2018 Dec 16];52(5):E173-9. Disponible en: https://www.ncbi.nlm.nih.gov/pmc /articles/PMC2769131/

14. Volpicelli G, Elbarbary $M$, Blaivas M, Lichtenstein DA, Mathis G, Kirkpatrick AW, et al. International evidence-based recommendations for point-of-care lung ultrasound. Intensive Care Med. 2012;38(4):577-91. https://doi.org/10. 1007/s00134-012-2513-4

15. Kaya Ş, Çevik AA, Acar N, Döner E, Sivrikoz C, Özkan R. A study on the evaluation of pneumothorax by imaging methods in patients presenting to the emergency department for blunt thoracic trauma. Ulus Travma Acil Cerrahi Derg. 2015 Sep;21(5):366-72. https://doi.org/10.5 505/tjtes.2015.91650

16. Reynolds TA, Amato S, Kulola I, Chen CJ, Mfinanga J, Sawe HR. Impact of point-of-care ultrasound on clinical decision-making at an urban emergency department in Tanzania. PLoS One. 2018 Apr 25;13(4):e0194774. https://doi.org/10. 1371/journal.pone.0194774

17. Udrea DS, Sumnicht A, Lo D, Villarreal L, Gondra S, Chyan R, et al. Effects of student-performed point-ofcare ultrasound on physician diagnosis and management of patients in the emergency department. J Emerg Med. 
2017;53(1):102-9. https://doi.org/10.1

016/j.jemermed.2017.01.021

18. Kline JP, Dionisio D, Suliivan

K, Early T, Wolf J, Kline

D. Detection of pneumothorax with ultrasound-ProQuest. AANA

J [internet]. 2013;81(4):265-72.

Disponible en: https://www.twinoaksa nesthesia.com/detection-of-pneumoth orax-with-ultrasound

19. Murphy M, Nagdev A, Sisson C. Lack of lung sliding on ultrasound does not always indicate a pneumothorax. Resuscitation. 2008;77(2):270.

20. Staub LJ, Rodolfo R, Biscaro M, Kaszubowski E, Maurici R. Chest ultrasonography for the emergency diagnosis of traumatic pneumothorax and haemothorax: a systematic review and meta-analysis. Int J Care Inj. 2018;49:457-66. https://doi.org/10.101 6/j.injury.2018.01.033

\section{Notas}

Conflicto de intereses Los autores declaran que no existe afiliación alguna con cualquier organización con un interés monetario o ético directo o indirecto con la sustancia de lo tratado en este escrito científico; por lo que todos los autores declaran que no existe conflicto de interés alguno que afecte el diseño y el reporte de los resultados del estudio. 